\title{
RANDOMIZED DOUBLE-BLIND CONTROLLED TRIAL TO EVALUATE EFFICACY OF VITAMIN D SUPPLEMENTATION AMONG PATIENTS WITH SYSTEMIC LUPUS ERYTHEMATOSUS
}

\author{
Wiyanoot Lomarat, Pongthorn Narongroeknawin, Sumapa Chaiamnuay, Paijit Asavatanabodee, \\ Rattapol Pakchotanon
}

\author{
Rheumatic Disease Unit, Department of Internal Medicine, Phramongkutklao Hospital and \\ College of Medicine, Bangkok, Thailand
}

\begin{abstract}
Objective/background: Patients with systemic lupus erythematosus (SLE) have a high prevalence of vitamin D deficiency. We aimed to assess the efficacy and safety of ergocalciferol combined with standard care among patients with SLE.

Methods: A randomized, double-blinded, placebo-controlled study was conducted among patients with SLE $(\mathrm{N}=104)$. The patients were randomized to receive either a higher dosage of ergocalciferol $(100,000 \mathrm{IU}$ of ergocalciferol weekly for 4 weeks followed by 40,000 IU of ergocalciferol weekly for 20 weeks, group $\mathrm{A}(\mathrm{N}=52)$ or placebo (group $\mathrm{B}, \mathrm{N}=52$ ). All patients received 800 units of cholecalciferol daily for 24 weeks. Concurrent medications were adjusted as clinically required. We compared demographics, serum 25-Hydroxy vitamin D $(25(\mathrm{OH}) \mathrm{D})$ levels, SLE disease activity index (SLEDAI-2K) and treatment variables between the two groups. The outcomes were measured at baseline, 12 and 24 weeks follow-up. These outcomes included serum 25(OH) D, SLEDAI-2K, SLE flare event defined by an increase of SLEDAI-2K between 2 visits, erythrocyte sedimentation rate (ESR), C-reactive protein (CRP), urine protein creatinine ratio (UPCR), health assessment questionnaire (HAQ), the change of dosage of immunosuppressive drugs and glucocorticoids (GCS) and ergocalciferol related toxicity. Subgroup analyses were also undertaken among patients with lupus nephritis. Continuous variables between the 2 groups were compared using student's t-test whereas categorical groups were compared using the chi-square test.

Results: Of 104 patients, only 88 patients completed the study. Baseline characteristics between the 2 groups were similar. At 24 weeks, the mean \pm standard difference (SD) of serum levels of $25(\mathrm{OH})$ $\mathrm{D}$ in group A was significantly higher than those in group B $(41.2 \pm 14.4$ vs. $27.2 \pm 10.1, p<0.001)$. No difference was observed between groups A and B with respect to SLEDAI-2K, flare event, ESR, CRP and dosage of immunosuppressive drugs. However, at 12 and 24 weeks, the number of patients who could reduce GCS dosage in group A were significantly greater than group B (at 12 weeks, 39.6 vs. $17.6 \%, p=0.008$; at 24 weeks, 43.4 vs. $23.5 \%, p=0.013$ ). Subgroup analysis revealed no significant improvement of UPCR in group A compared with group B. Ergocalciferol related adverse reactions in both groups were similar. Serum calcium levels did not change within and between groups of treatment. Conclusion: This study was inconclusive in demonstrating the efficacy of high dose ergocalciferol in controlling SLE disease activity. However, high dose ergocalciferol could be a safe adjunctive therapy that has a corticosteroid-sparing effect on patients with SLE.
\end{abstract}

Keywords: Vitamin D level, Systemic Lupus Erythematosus

J Southeast Asian Med Res 2020; 4(1):24-32

http://www.jseamed.org

Correspondence: Pakchotanon R., Rheumatic Disease Unit, Department of Internal Medicine, Phramongkutklao Hospital and College of Medicine

315 Ratchawithi Rd., Ratchathewi, Bangkok, Thailand 10400

Tel: +662-354-7980 (Office) Fax +662-354-7980 (Office)

E-mail: rattapolpmk@gmail.com

Received: 24 November 2019, Revised: 7 April 2020, Accepted: 12 May 2020 


\section{Introduction}

Vitamin D is a secosteroid that carries a structure similar to a steroid. The main source of vitamin D is de novo synthesis in the skin through ultraviolet irradiation of 7 dehydrocholesterol. Vitamin D is related to mineral metabolism and skeletal health. Vitamin D enhances intestinal calcium, phosphate absorption stimulated osteoclast differentiation, calcium resorption from bone and promotes mineralization of bone matrix. ${ }^{(1,2)}$

Over the last decade, several studies found vitamin D receptor (VDR) and vitamin D activating enzyme 1, $\alpha$-hydroxylase (CYP27B1) expressed in many cell types especially cells of the immune system. Vitamin D deficiency has effects on innate and adaptive immune systems and possibly enhances the activity or occurrence of autoimmune disease. ${ }^{(3-6)}$ It has been shown that 1 , 25-dihydrovitamin D3 could inhibit dendritic cell maturation and expression of IFN- $\alpha$ gene among patients with Systemic Lupus Erythematosus (SLE). ${ }^{(7)}$ A recent study also demonstrated patients with vitamin D-deficient SLE had higher serum IFN- $\alpha$ activity and B-cell activation compared with those patients with higher vitamin D levels. ${ }^{(8)}$

Several studies demonstrated a high prevalence of vitamin D deficiency among patients with SLE due to multiple risk factors such as sunlight avoidance, glucocorticoids and hydroxychloroquine exposure and chronic kidney disease. ${ }^{(9)}$ Low serum vitamin D levels have been suspected as a risk factor in developing SLE, persistence of disease activity and increasing morbidity and mortality among patients with SLE (10-12). A recent study among Thai patients with SLE demonstrated a statistically significant inverse relationship between disease activity scores of SLE and serum levels of 25-hydroxyvitamin D and inverse relationship between urine creatinine ratio and serum levels of 25-hydroxyvitamin D. $(13,14)$ Two recent studies evaluating the efficacy of vitamin D supplementation among patients with SLE found that high dose vitamin D supplementation increased naive CD4 $+\mathrm{T}$ cells, Tregs and decreased effector Th1 and Th17 cells, memory B cells and anti-DNA antibodies. Another study also showed significantly improved levels of pro-inflammatory cytokines and hemostatic markers (fibrinogen, von Willebrand factor). ${ }^{(15,16)}$
This study aimed to assess the efficacy and safety of high dose ergocalciferol combined with standard care among patients with SLE by measuring the level of 25-hydroxyvitamin D, an inflammatory marker, disease activity index, quality of life, adverse events and concurrent medications.

\section{Methods}

Study Design

A randomized double-blind controlled trial was conducted in the Rheumatic Department, Phramongkutklao Hospital. The study was approved by the Institutional Review Board of the Royal Thai Army Medical Department. All subjects provided informed consent and all procedures were performed under the ethics standard of the responsible committee on human experimentation. Following informed consent, eligibility criteria and clinical status were assessed at first visit.

Subjects were enrolled from November 2013 to January 2014. All subjects fulfilled at least 4 of the American College of Rheumatology (ACR) classification criteria for SLE, 1997. Inclusion criteria included patients with SLE at least 18 years of age. Female subjects were eligible to enter the study if they were not pregnant or nursing. All subjects had the ability to understand the requirements of the study to provide written informed consent and complied with the study protocol procedures. Subjects were excluded if they had other co-existing auto-immune diseases (except for secondary Sjogren's Syndrome), history of liver disease, history of renal stone, chronic inflammatory or infectious condition, active cancer or laboratory abnormality of the following conditions, i.e., serum creatinine $>2.5 \mathrm{~g} / \mathrm{dl}$, serum calcium $>10.5 \mathrm{mg} / \mathrm{dl}$ or serum $25(\mathrm{OH}) \mathrm{D}<10 \mathrm{ng} / \mathrm{ml}$.

All subjects and physicians were blinded to group assignment and treatment allocation. Subjects were randomized in 2 parallel groups, randomized by box of 4 using computer analysis at baseline of treatment. All subjects gave a detailed history and received a thorough physical examination. Clinical data recorded included disease duration, body mass index, medication use including mean daily dosage of corticosteroid, antimalarial and other immunosuppressive drugs. Subjects were randomized in 2 groups. The high dose (HD) group was assigned to receive 100,000 IU of 
ergocalciferol weekly for 4 weeks followed by 40,000 IU of ergocalciferol weekly for 20 weeks. The placebo group received identical placebo 24 weeks and both groups received 800 units of cholecalciferol daily for 24 weeks. Both groups were allowed to adjust the dose of corticosteroid, antimalarial and immunosuppressive drugs followed by disease activity.

\section{$25(\mathrm{OH})$ vitamin $D$ level measurement and definitions}

Serum samples for $25(\mathrm{OH})$ vitamin D levels were obtained from all randomized subjects before administering ergocalciferol or placebo at the baseline visit and end of study (week 24). The $25(\mathrm{OH})$ vitamin D level was measured using a chemiluminescence immunoassay. In accordance with the US Endocrine Society, vitamin D deficiency was defined as $25(\mathrm{OH})$ vitamin D level of $20 \mathrm{ng} / \mathrm{ml}$ or less, vitamin D insufficiency as 21 to $29 \mathrm{ng} / \mathrm{ml}$, and vitamin D sufficiency as $30 \mathrm{ng} / \mathrm{ml}$ or higher. ${ }^{(9,16,17)}$ Biological markers and auto-antibodies measurements included complete bloodcount, erythrocyte sedimentation rate(ESR), blood urea nitrogen, creatinine, liver function test, serum calcium and phosphorus levels, C-reactive protein (CRP), autoantibodies levels (anti-dsDNA), C3, C4, lupus anticoagulant, serum anti-cardiolipin and $\mathrm{B} 2 \mathrm{glycop}$ rotein. Urinary analysis included urine protein-creatinine ratio (UPCR). These biomarkers were measured at baseline and at weeks 12 and 24.

Disease activity assessments on SLE Disease activity index (SLEDAI) and SLE flare index were evaluated at baseline, weeks 12 and 24. Quality of life was evaluated at baseline and end of the study. Treatment-related adverse events reported by patients or observed by physicians were collected at every visit.

\section{Efficacy endpoints and analysis}

Primary efficacy endpoints included the change of inflammatory and disease activity markers (ESR,
CRP, anti-dsDNA, C3, C4, lupus anticoagulant, serum anti-cardiolipin, B2glycoprotein) and disease activity index (SLEDAI, HAQ) at week 12 and the end of study. Secondary efficacy endpoints included the renal response in one patient with lupus nephritis at the end of the study. Lupus nephritis was determined by UPCR $\geq 0.5$ and the renal response was determined by changes in proteinuria (UPCR below 0.5 and/or 25 to $50 \%$ reduced proteinuria) and the dose adjustment of prednisolone and other immunosuppressive drugs.

\section{Statistical analysis}

Data were analyzed using SPSS Software, Version 20. Values in the study were expressed as mean (SD), median (IQR) or number (percentage). Continuous variables between the 2 groups were compared using the student's t-test, and categorical groups were compared using the chi-square test. Statistical significance was defined as a 2-sided $p$-value of 0.05 .

\section{Results}

The 104 eligible participants (91 woman, 13 men) were randomized in either high dose group (HD-groups, $n=52$ ) or placebo group $(n=52)$. The mean age was 41.15 years in the high dose group and 43.67 years in the placebo group. Eighty-five patients (81\%) completed the protocol at the end of 24 weeks of treatments.-

The demographic characteristics of study groups are depicted in Table 1. Baseline characteristics were similarly distributed between the 2 treatment groups except only hypertension was more common in the placebo group. Mean $25(\mathrm{OH})$ $\mathrm{D}$ levels, inflammatory, disease activity marker, renal response of vitamin D supplement of the study population at baseline and 24 weeks of treatment are shown in Table 2. 
Table1. Baseline characteristics of study participants according to treatment groups

\begin{tabular}{|c|c|c|c|}
\hline & High dose group & Placebo group & $p$-value \\
\hline Sex, female/male, $(n, \%)$ & $48(90.57 \%)$ & $43(84.31 \%)$ & 0.386 \\
\hline Age, years, mean (SD) & $41.15(13.31)$ & $43.67(13.19)$ & 0.336 \\
\hline BMI, kg/m2, mean (SD) & $22.01(5.49)$ & $22.59(5.59)$ & 0.533 \\
\hline DM (n, \%) & $7(13.21 \%)$ & $4(7.84 \%)$ & 0.374 \\
\hline HT (n, \%) & $18(33.96 \%)$ & $28(54.90 \%)$ & 0.032 \\
\hline DLD (n, \%) & $14(26.42 \%)$ & $22(43.14 \%)$ & 0.073 \\
\hline CAD $(n, \%)$ & $3(5.56 \%)$ & $3(5.58 \%)$ & 0.961 \\
\hline Hypothyroid (n, \%) & $2(3.77 \%)$ & 0 & 0.161 \\
\hline $\operatorname{APS}(n, \%)$ & $4(7.55 \%)$ & $6(11.76 \%)$ & 0.466 \\
\hline Disease duration (months), median (IQR) & $72(24,144)$ & $120(48,228)$ & 0.052 \\
\hline Vitamin D Supplement IU/day, median (IQR) & $800(800,2857)$ & $800(600,2875)$ & 0.632 \\
\hline Drug calcium (mg), mean (SD) & $1301.89(512.72)$ & $1264.71(550.94)$ & 1.0 \\
\hline Prednisolone (mg / day), median (IQR) & $8.75(5,15)$ & $5(2.5,10)$ & 0.257 \\
\hline CQ (mg/day), mean (SD) & $207.63(65.16)$ & $197.5(73)$ & 0.669 \\
\hline HCQ (mg/day), mean (SD) & $180(40.82)$ & $193.8(102.6)$ & 0.538 \\
\hline Azathioprine (mg/day), mean (SD) & $55.25(26.73)$ & $59.1(23.1)$ & 0.699 \\
\hline CYC iv (mg/month), mean (SD) & $1000(200)$ & $800(163.3)$ & 0.203 \\
\hline Cyclosporin A (mg/day), mean (SD) & - & 100 & NA \\
\hline MTX (mg/week), mean (SD) & $6.25(1.77)$ & - & NA \\
\hline MMF (mg/day), mean (SD) & $1416(664.58)$ & $1647(852.2)$ & 0.609 \\
\hline SLEDAI, median (IQR) & $4.0(2,6)$ & $4.0(1,4)$ & 0.544 \\
\hline c3 (g/l), mean (SD) & $0.81(0.34)$ & $0.83(0.34)$ & 0.757 \\
\hline c4 (g/l), mean (SD) & $0.20(0.13)$ & $0.19(0.11)$ & 0.869 \\
\hline Anti-dsDNA (U/ml), median (IQR) & $135(25.9,408.5)$ & $142.25(29.43,472)$ & 0.797 \\
\hline ESR (mm/hour), mean (SD) & $49.17(26.47)$ & $41.89(21.62)$ & 0.150 \\
\hline CRP (mm/hour), median (IQR) & $1.6(0.6,3)$ & $1.4(0.6,3.3)$ & 0.892 \\
\hline $25(\mathrm{OH}) \mathrm{D}(\mathrm{ng} / \mathrm{ml})$, mean (SD) & $27.99(9.24)$ & $25.92(10.57)$ & 0.288 \\
\hline Ca (mg/dl), mean (SD) & $9.16(0.49)$ & $9.07(0.52)$ & 0.396 \\
\hline po4 (mg/dl), mean (SD) & $3.68(0.6)$ & $3.57(0.66)$ & 0.359 \\
\hline Alb (mg/ml), mean (SD) & $4.06(0.4)$ & $4.12(0.5)$ & 0.520 \\
\hline UPCR, mean (SD) & $0.35(0.65)$ & $0.47(0.99)$ & 0.470 \\
\hline Cardiolipin (U/ml), median (IQR) & $1.8(0.85,2.86)$ & $1.3(0.7,3.6)$ & 0.479 \\
\hline b2gly (U/ml), median (IQR) & $9.2(4.4,14.25)$ & $7.9(4.5,12)$ & 0.734 \\
\hline HAQ, mean (SD) & $64.42(20.93)$ & $68.12(33.8)$ & 0.502 \\
\hline
\end{tabular}

DLD: dyslipidemia; CAD: coronary artery disease; SLEDAI: Systemic Lupus Erythematosus Disease Activity Index; CQ: chloroquine; HCQ: hydroxychloroquine; MTX: methotrexate; CYC: cyclophosphamide; MMF: mycophenolate mofetil; Alb: serum albumin; UPCR: urine protein-creatinine ratio; HAQ: health assessment questionnaire. 
Table 2. Vitamin D status and inflammatory and disease activity markers at baseline and 24 weeks of treatment

\begin{tabular}{|c|c|c|c|}
\hline & High dose group & Placebo group & $p$-value \\
\hline \multicolumn{4}{|l|}{ Serum vitamin D (ng/ml) (mean, SD) } \\
\hline Baseline & $27.99(9.24)$ & $25.92(10.57)$ & 0.288 \\
\hline 24 weeks & $41.17(14.44)$ & $27.16(10.12)$ & $<0.001$ \\
\hline \multicolumn{4}{|l|}{ Anti-dsDNA (U/ml), median (IQR) } \\
\hline Baseline & $135(25.9,408.5)$ & $142.25(29.43,472)$ & 0.797 \\
\hline 24 weeks & $164.80(40.55,321.0)$ & $102.3(42.5,260)$ & 0.706 \\
\hline \multicolumn{4}{|l|}{ C3 $(g, l)$ (mean, SD) } \\
\hline Baseline & $0.81(0.34)$ & $0.83(0.34)$ & 0.757 \\
\hline 24 weeks & $0.96(0.35)$ & $0.92(0.36)$ & 0.606 \\
\hline \multicolumn{4}{|l|}{ C4 (g, l) (mean, SD) } \\
\hline Baseline & $0.20(0.13)$ & $0.19(0.11)$ & 0.869 \\
\hline 24 weeks & $0.20(0.11)$ & $0.27(0.28)$ & 0.166 \\
\hline \multicolumn{4}{|l|}{$\operatorname{ESR}(\mathbf{m m}, \mathrm{hr})($ mean, SD) } \\
\hline Baseline & $49.17(26.47)$ & $41.89(21.26)$ & 0.150 \\
\hline 24 weeks & $45.55(25.99)$ & $39.17(20.27)$ & 0.219 \\
\hline \multicolumn{4}{|l|}{ CRP (mm, hr) (median) (IQR) } \\
\hline Baseline & $1.6(0.6,3)$ & $1.4(0.6,3.3)$ & 0.892 \\
\hline 24 weeks & $0.9(0.5,2.0)$ & $1.4(0.55,2.9)$ & 0.442 \\
\hline \multicolumn{4}{|l|}{ Cardiolipin (U/ml) (median) (IQR) } \\
\hline Baseline & $1.8(0.85,2.86)$ & $1.3(0.7,3.6)$ & 0.479 \\
\hline 24 weeks & $1.1(0.8,2.5)$ & $1.7(0.8,2.6)$ & 0.284 \\
\hline \multicolumn{4}{|l|}{ B2 glycoprotein (U/ml) (median) (IQR) } \\
\hline Baseline & $9.2(4.4,14.25)$ & $7.9(4.5,12)$ & 0.734 \\
\hline 24 weeks & $8.4(4.6,18.2)$ & $8.1(3,14)$ & 0.478 \\
\hline $\begin{array}{l}\text { Subgroup of subjects with } \mathrm{UPCR} \geq 0.5 \\
\text { at baseline }\end{array}$ & $\mathrm{N}=36$ & $\mathrm{~N}=33$ & \\
\hline \multicolumn{4}{|l|}{ UPCR (mean) (SD) } \\
\hline UPCR at baseline & $1.28(1.07)$ & $2.67(1.29)$ & 0.028 \\
\hline UPCR at 24 weeks & $0.83(0.82)$ & $0.55(0.65)$ & 0.561 \\
\hline \multicolumn{4}{|l|}{ Renal response $(\mathrm{N}, \%)$} \\
\hline $\begin{array}{l}25 \% \text { decreased UPCR or UPCR }<0.5 \\
\text { at } 24 \text { weeks }\end{array}$ & $19(57.6 \%)$ & $11(30.6 \%)$ & 0.024 \\
\hline $\begin{array}{l}50 \% \text { decreased UPCR or UPCR }<0.5 \\
\text { at } 24 \text { weeks }\end{array}$ & $11(33.3 \%)$ & $8(22.2 \%)$ & 0.302 \\
\hline
\end{tabular}

UPCR: urine protein-creatinine ratio.

Vitamin D status

Vitamin D status of the HD-group at baseline was similar to the placebo group $(27.99 \pm 9.24$ vs. $25.92 \pm 10.57 \mathrm{ng} / \mathrm{ml}, \mathrm{p}=0.288$ ). At baseline, a small number of patients in the HD-group had vitamin D deficiency $(<20 \mathrm{ng} / \mathrm{ml})$ compared with the placebo group $(20.75$ vs. $33.33 \%, \mathrm{p}=0.148)$. At the end of the study, mean $25(\mathrm{OH}) \mathrm{D}$ levels of the HD-group were significantly higher than 
those of the placebo group $(41.2 \pm 14.4$ vs. 27.2 $\pm 10.1, p<0.001)$. In addition, a significantly lower number of subjects in the HD group had vitamin $\mathrm{D}$ insufficiency than that of the placebo group ( 7.55 vs. $21.57 \%, P=0.035)$ at 24 weeks of treatment.

\section{Inflammatory and disease activity markers}

Inflammatory and disease activity markers including anti-dsDNA, C3, C4, CRP, ESR, B2-glycoprotein and anti-cardiolipin were similar between the HD and placebo groups during the study. Subgroup analysis was performed among subjects with $25(\mathrm{OH})$ D levels below $30 \mathrm{ng} / \mathrm{ml}$ at baseline. No significantly improved inflammatory and disease activity markers were found between the two treatment groups (data not shown).

\section{Subgroup of patients with lupus nephritis}

In all, 17 patients presented lupus nephritis at baseline (HD-group, $\mathrm{n}=10$ and placebo group, $\mathrm{n}=7$ ). Renal response among patients with lupus nephritis at the end of the study between the two groups is shown in Table 2. At baseline, the mean level of UPCR in the HD-group was significantly lower than that of the placebo group $(1.28 \pm 1.07$ vs. $2.67 \pm 1.29, \mathrm{p}=0.028$ ). At 24 weeks of treatment, no significant difference was observed in the mean level of UPCR between two groups $(0.83 \pm 0.82$ vs. $0.55 \pm 0.65)$. However, the number of subjects presenting lupus nephritis with $25 \%$ reduced UPCR or UPCR below $500 \mathrm{mg} /$ day and the number of subjects with lupus nephritis with $50 \%$ reduced UPCR or UPCR below $500 \mathrm{mg} /$ day did not significantly differ between two groups.

\section{Disease activity index}

Efficacies of vitamin D supplement are shown in Table 3. In this study, no significant improvement was shown in both disease activity indexes (SLEDAI, flare index) and quality of life determined using a health assessment questionnaire (HAQ) between the two study groups.

Table 3. Variations in SLEDAI score, HAQ score and number of patients (n, \%) with an improved flare index in the HD-group compared with those of the placebo group, values are mean (SD), median (IQR)

\begin{tabular}{lccc}
\hline \multicolumn{1}{c}{ SLEDAI Median (IQR) } & High dose group & Placebo group & $\boldsymbol{p}$-value \\
\hline At baseline & $4.0(2,6)$ & $4.0(1,4)$ & 0.544 \\
At 12 weeks of treatment & $2.0(0,4)$ & $2.0(0,2)$ & 0.880 \\
At 24 weeks of treatment & $0.0(0,4)$ & $2.0(0,2)$ & 0.101 \\
HAQ (mean, SD) & & & \\
At baseline & $64.42(20.93)$ & $68.12(33.8)$ & 0.502 \\
At 24 weeks of treatment & $60.04(22.42)$ & $58.09(21.3)$ & 0.672 \\
SLE not flare (N, \%) & $39(73.6 \%)$ & $42(82.4 \%)$ & 0.266 \\
At 24 weeks of treatment & & & \\
\hline
\end{tabular}

SLEDAI: Systemic Lupus Erythematosus Disease Activity Index; UPCR: urine protein-creatinine ratio; HAQ: health assessment questionnaire.

\section{Vitamin D as steroid-sparing effect and effect on immunosuppressant}

Significantly more subjects could reduce the dose of prednisolone in the HD group compared with the placebo group at 12 weeks until the end of the study (12 weeks; $43.75 \%$ vs. $18.75 \%$, $p=0.008$ and 24 weeks; $52.27 \%$ vs. $26.66 \%$, $p=0.024)$ and at 6 months of treatment $(52.27 \%$ vs. $26.66 \%, p=0.024 *)$. In addition, significant difference was found in the dose adjustment of immunosuppressive drugs between the two groups during the study. Data are shown in Table 4. 
Table 4. Number of subjects with reduced prednisolone and immunosuppressive doses at 12 and 24 weeks of treatment.

\begin{tabular}{llll}
\hline $\begin{array}{l}\text { The number of subjects with a reduced } \\
\text { prednisolone dose }\end{array}$ & High dose group & Placebo group & $\boldsymbol{p}$-value \\
\hline $\begin{array}{l}\text { At } 12 \text { weeks of treatment (n, \%) } \\
\text { At } 24 \text { weeks of treatment (n, \%) }\end{array}$ & $21(39.62 \%)$ & $9(17.65 \%)$ & 0.008 \\
$\begin{array}{l}\text { The number of subjects with reduced } \\
\text { immunosuppressive dose }\end{array}$ & $23(43.40 \%)$ & $12(23.53 \%)$ & 0.013 \\
At 12 weeks of treatment (n, \%) & & \\
At 24 weeks of treatment (n, \%) & $2(3.8 \%)$ & 0 & 0.242 \\
\hline
\end{tabular}

\section{Safety profile}

One patient in the HD group withdrew from the study due to minor rash reaction and recovered after vitamin D discontinuation. One patient in the placebo group withdrew from the study due to weight gain. Serum calcium and phosphorus levels did not change within and between groups of treatment (data not shown).

\section{Discussion}

In the view of the potential beneficial effects of vitamin $\mathrm{D}$ on immunomodulation and high prevalence of vitamin D deficiency among patients with $\operatorname{SLE}^{(8,10,16,18,19)}$, supplementation of vitamin $\mathrm{D}$ constitutes an important therapeutic strategy. Avoidance of sunlight, renal insufficiency, obesity, and use of medication such as glucocorticoids are major risk factors for vitamin D insufficiency in SLE. (14) This study showed the effect of high dose over low dose vitamin D supplementation on rising serum vitamin D levels among patients with SLE.

The immunomodulation of vitamin D is opposite that of the observed immunological aberrations among patients with SLE. A related study demonstrated that 1, 25-dihydroxy vitamin D and its analogs inhibited polyclonal and antidsDNA ( $\mathrm{IgG}$ ) production by stimulating peripheral blood mononuclear cells from patients with SLE. ${ }^{(20)}$ Dihydroxy vitamin D3 has been shown to inhibit dendritic cell maturation and expression of the IFN- $\alpha$ gene (IFN signature) among patients with SLE. ${ }^{(1,7,25)}$ A more recent study also demonstrated that vitamin D deficient patients with SLE had higher serum IFN- $\alpha$ activity and B-cell activation compared with those patients with higher vitamin D levels taken together. Strong evidence indicates that insufficient vitamin D may aggravate immunological abnormalities among patients with SLE ${ }^{(8)}$ Several studies have reported an inverse relationship exists between $25(\mathrm{OH}) \mathrm{D}$ level and disease activity. ${ }^{(16,21,22)}$ Abou-Raya et al. showed that high dose vitamin D supplementation provided a significant effect on the reducing autoantibodies levels (anti-sm, anti-dsDNA) and ESR with improved complement level and SLEDAI score. ${ }^{(16)}$ This study could not demonstrate improved inflammatory markers, immunological markers, disease activity or quality of life of patients with SLE. This could be explained by the small sample size, short duration of follow-up, a small number of patients with vitamin D deficiency and uncontrolled concurrent medications.-

Regarding the subgroup analysis of patients with lupus nephritis, this study found no significantly improved UPCR among patients receiving high dose of vitamin D supplementation. According to a recent study of Petri $\mathrm{M}$ el al., vitamin $\mathrm{D}$ supplementation among patients with SLE with low level $25(\mathrm{OH}) \mathrm{D}(<40 \mathrm{ng} / \mathrm{ml})$ and $20 \mathrm{ng} /$ $\mathrm{ml}$ increase in $25(\mathrm{OH}) \mathrm{D}$ level were associated with a $21 \%$ decrease in the odds of having a high disease activity score and $15 \%$ decrease in the odds of having clinically improved proteinuria. ${ }^{(23)}$ This could be explained by the small number of patients with lupus nephritis as well as short duration of follow-up.

Interestingly, our study was the first to find a steroid-sparing effect of high-dose vitamin D supplementation. A significantly improved de-escalating of corticosteroid among patients receiving high dose vitamin $\mathrm{D}$ supplementation was observed, despite no differences in adjusting immunosuppressive agents. Two cross-sectional studies found an inverse relationship between levels of vitamin D and corticosteroid use (both 
current dose and cumulative dose) which might have supported corticosteroid-sparing effects in our study $(14,24)$. However, a future study controlling the dosage of immunosuppression is needed to confirm these findings.

The long term use of vitamin D is not completely safe, concerning hypercalcemia, hyperphosphatemia and renal stones, but the risks are extremely low. Long term follow-up is needed to ensure sufficient levels of $25(\mathrm{OH}) \mathrm{D}$ leads to clinically improved disease activity. Finally, we recommend a routine assessment of vitamin D levels and adequate supplementation of vitamin D among patients with SLE.

\section{Conclusion}

This study could not demonstrate the efficacy of high dose ergocalciferol in controlling SLE disease activity. However, high dose ergocalciferol could improve vitamin D levels back to normal and constitute a safe adjunctive therapy exhibiting a steroid-sparing effect among patients with SLE.

\section{Acknowledgments}

The authors would like to thank Ms Dollapas Punpanich for statistical analysis of the study. This study was supported by a grant from the Thai Rheumatism Association.

\section{Disclosures None}

\section{References}

1. Pike JW, Meyer MB. The vitamin D receptor: new paradigms for the regulation of gene expression by 1,25-dihydroxyvitamin $\mathrm{D}(3)$. Endocrinol Metab Clin North Am 2010; 39: 255-69.

2. Adams JS, Hewison M. Update in vitamin D. J Clin Endocrinol Metab 2010; 95: 471-8.

3. Hewison M. Vitamin D and the immune system: new perspectives on an old theme. Endocrinol Metab Clin North Am. 2010; 39: 365-79.

4. Adorini L, Penna G. Control of autoimmune diseases by the vitamin $\mathrm{D}$ endocrine system. Nat Clin Pract Rheumatol 2008; 4: 404-12.

5. Baeke F, Takiishi T, Korf H, Gysemans C, Mathieu C. Vitamin D: modulator of the immune system. Curr Opin Pharmacol 2010; 10: 482-96.
6. Chen S, Sims GP, Chen XX, Gu YY, Chen S, Lipsky PE. Modulatory effects of 1 , 25-dihydroxyvitamin D3 on human B cell differentiation. J Immunol 2007; 179: 1634-47.

7. Ben-Zvi I, Aranow C, Mackay M, Stanevsky A, Kamen DL, Marinescu LM, et al. The impact of vitamin D on dendritic cell function in patients with systemic lupus erythematosus. PloS One. 2010; 5: e9193.

8. Ritterhouse LL, Crowe SR, Niewold TB, Kamen DL, Macwana SR, Roberts VC, et al. Vitamin D deficiency is associated with an increased autoimmune response in healthy individuals and in patients with systemic lupus erythematosus. Ann Rheum Dis 2011; 70: 1569-74.

9. Singh A, Kamen DL. Potential benefits of vitamin D for patients with systemic lupus erythematosus. Dermatoendocrinol 2012; 4: 146-51.

10. Kamen DL, Cooper GS, Bouali H, Shaftman SR, Hollis BW, Gilkeson GS. Vitamin D deficiency in systemic lupus erythematosus. Autoimmun Rev 2006; 5: 114-7.

11. Hamza RT, Awwad KS, Ali MK, Hamed AI. Reduced serum concentrations of 25-hydroxy vitamin D in Egyptian patients with systemic lupus erythematosus: relation to disease activity. Medical science monitor : Int Med J Ex Clin Res 2011; 17: CR711-8.

12. Cutillas-Marco E, Morales-Suarez-Varela M, Marquina-Vila A, Grant W. Serum 25-hydroxy vitamin D levels in patients with cutaneous lupus erythematosus in a Mediterranean region. Lupus 2010; 19: 810-4.

13. Sumethkul K, Boonyaratavej S, Kitumnuaypong T, Angthararuk S, Cheewasat P, Manadee N, etal. The predictive factors of low serum 25hydroxy vitamin Dand vitamin D deficiency in patients with systemic lupus erythematosus. Rheumatol Int 2013; 33: 1461-7.

14. Chaiamnuay S, Chailurkit LO,Narongroeknawin P, Asavatanabodee P, Laohajaroensombat S, Chaiamnuay P. Current daily glucocorticoid use and serum creatinine levels are associated with lower $25(\mathrm{OH})$ vitamin D levels in Thai patients with systemic lupus erythematosus. J Clin Rheumatol 2013; 19: 121-5.

15. Terrier B, Derian N, Schoindre Y, Chaara W, Geri G, Zahr N, et al. Restoration of regulatory 
and effector $\mathrm{T}$ cell balance and $\mathrm{B}$ cell homeostasis in systemic lupus erythematosus patients through vitamin D supplementation. Arthritis Res Ther 2012; 14: R221.

16. Abou-Raya A, Abou-Raya S, Helmii M. The effect of vitamin D supplementation on inflammatory and hemostatic markers and disease activity in patients with systemic lupus erythematosus: a randomized place bocontrolled trial. Journal Rheumatol 2013; 40: 265-72.

17. Hochberg MC. Updating the American College of Rheumatology revised criteria for the classification of systemic lupus erythematosus. Arthritis Rheum 1997; 40: 1725.

18. Pelajo CF, Lopez-Benitez JM, Miller LC. Vitamin D and autoimmune rheumatologic disorders. Autoimmun Rev 2010; 9: 507-10.

19. Gatenby P, Lucas R, Swaminathan A. Vitamin D deficiency and risk for rheumatic diseases: an update. Curr Opin Rheumatol 2013; 25: 184-91.

20. Linker-Israeli M, Elstner E, Klinenberg JR, Wallace DJ, Koeffler HP. Vitamin D(3) and its synthetic analogs inhibit the spontaneous in vitro immunoglobulin production by SLE-derived PBMC. Clin Immunol 2001; 99: 82-93.

21. Bonakdar ZS, Jahanshahifar L, Jahanshahifar F, Gholamrezaei A. Vitamin D deficiency and its association with disease activity in new cases of systemic lupus erythematosus. Lupus 2011; 20: 1155-60.

22. Costenbader KH, Feskanich D, Holmes M, Karlson EW, Benito-Garcia E. Vitamin D intake and risks of systemic lupus erythematosus and rheumatoid arthritis in women. Ann Rheum Dis 2008; 67: 530-5.

23. Petri M, Bello KJ, Fang H, Magder LS. Vitamin D in systemic lupus erythematosus: modest association with disease activity and the urine protein-to-creatinine ratio. Arthritis Rheum 2013; 65: 1865-71.

24. Toloza SM, Cole DE, Gladman DD, Ibanez D, Urowitz MB. Vitamin D insufficiency in a large female SLE cohort. Lupus 2010; 19: 13-9. 